\title{
Clinical, pathological and genetic features of anaplastic and poorly differentiated thyroid cancer: A single institute experience
}

\author{
CRISTINA ROMEI $^{1}$, ALESSIA TACITO ${ }^{1}$, ELEONORA MOLINARO ${ }^{1}$, PAOLO PIAGGI $^{1}$, \\ VIRGINIA CAPPAGLI ${ }^{1}$, LETIZIA PIERUZZI ${ }^{1}$, ANTONIO MATRONE ${ }^{1}$, DAVID VIOLA $^{1}$, LAURA AGATE ${ }^{1}$, \\ LIBORIO TORREGROSSA ${ }^{2}$, CLARA UGOLINI ${ }^{2}$, FULVIO BASOLO ${ }^{2}$, LUIGI DE NAPOLI ${ }^{2}$, \\ MICHELE CURCIO $^{3}$, RAFFAELE CIAMPI ${ }^{1}$, GABRIELE MATERAZZI ${ }^{2}$, PAOLO VITTI ${ }^{1}$ and ROSSELLA ELISEI ${ }^{1}$ \\ ${ }^{1}$ Endocrine Unit, Department of Clinical and Experimental Medicine; ${ }^{2}$ Department of Surgical, Medical and \\ Molecular Pathology; ${ }^{3}$ Division of Transfusion Medicine and Transplant Biology, \\ University Hospital of Pisa, I-56124 Pisa, Italy
}

Received September 2, 2017; Accepted January 22, 2018

DOI: $10.3892 / \mathrm{ol} .2018 .8470$

\begin{abstract}
Anaplastic (ATC) and poorly differentiated thyroid cancer (PDTC) are very aggressive cancers whose histological diagnosis is not always straightforward. Clinical, pathological and genetic features may be useful to improve the identification of these rare histotypes. In the present study the clinical, pathological and genetic features of two groups of ATC $(n=21)$ and PDTC $(n=21)$ patients were analyzed. Clinical data were retrieved from a computerized database. The oncogenic profiles were studied using the Sanger sequencing method of a selected series of oncogenes and/or tumor suppressor genes known to be altered in these tumors. The presence of macrophages in both series of tissues was evaluated by immunohistochemistry. Patients with ATC were older and affected by a more advanced disease at diagnosis than those with PDTC. The median survival was significantly shorter in ATC compared with PDTC patients $(\mathrm{P}=0.0014)$. ATC showed a higher prevalence of TP53 and TERT mutations (10/21, $47.6 \%$ and $9 / 21,42.8 \%$, respectively) while $T E R T$ and $B R A F$ mutations were the most prevalent in the PDTC group (7/21, $33.3 \%$ and $4 / 23,19 \%$ respectively). Genetic heterogeneity (i.e., $>2$ mutations) was more frequent in $\operatorname{ATC}(10 / 21,28.6 \%)$ compared with in PDTC $(3 / 21,4.7 \%)(\mathrm{P}=0.03)$. Macrophages were more frequently present in ATC, particularly in those cases with TP53 mutations. In conclusion, these data indicate that ATC and PDTC may be characterized by different clinical, pathological and genetic profiles. In particular ATC, but not PDTC, were positive for TP53 and PTEN alterations. Complex mutations were also found in ATC but not in PDTC.
\end{abstract}

Correspondence to: Dr Rossella Elisei, Endocrine Unit, Department of Clinical and Experimental Medicine, University Hospital of Pisa, Via Paradisa, 2, I-56124 Pisa, Italy

E-mail: rossella.elisei@med.unipi.it

Key words: anaplastic thyroid cancer, poorly differentiated thyroid cancer, TP53, macrophages, TERT, PTEN
Moreover, genetic heterogeneity was more frequent in ATC than PDTC. Finally, TP53 mutation and the accumulation of several mutations correlated with a shorter survival time.

\section{Introduction}

Anaplastic (ATC) and poorly differentiated thyroid cancer (PDTC) take origin from the follicular cells but during the process of tumoral transformation, they lose progressively the typical features of these cells, being the ATC completely undifferentiated while PDTC showing an intermediate spectrum of differentiation $(1,2)$. These tumors, although rare (about 2 and 5\% of thyroid cancer cases, respectively) (3), represent the most aggressive thyroid tumors but their median survival time is rather different being of 6-12 months and 3-5 years for ATC and PDTC, respectively $(4,5)$.

The histological diagnosis of ATC and PDTC is not always straightforward (6) and some controversial issues regarding the differential diagnosis among ATC and PDTC are not yet fully resolved by pathologists (7). However, at least two or three histological classifications to better distinguish these two types of advanced thyroid tumors have been proposed and the one chosen by the pathologist for the diagnosis should be indicated in the pathological report $(8,9)$.

Molecular alterations causative of these tumors have been studied over the years by Sanger analysis and/or other techniques $(10,11)$. Very recently the genomic and transcriptomic landscape of ATC and PDTC has been studied with a Next Generation Sequencing (NGS) approach (12). This study demonstrated that ATC, and to a lesser extent PDTC, are characterized by the accumulation of several different oncogenic alterations. In particular, so far, no genetic mutations or chromosomal rearrangements have been demonstrated to be unique for ATC and/or PDTC but, it is worth to note, that the majority of these studies are affected by the lack of using common histologic criteria to define either ATC or PDTC (13). In both cases the two most frequently altered genes are TERT oncogene and TP53 tumor suppressor gene. However, while the prevalence of the mutations of the two genes is very high in ATC (i.e., 70-75\%) they are much lower, especially for 
TP53, in PDTC (i.e., 40 and 8\%, for TERT and TP53, respectively). Mutations of other genes that impair the MAPK and PI3K-AKT pathways including BRAF, RAS, AKT, PTEN and $P I K 3 C A$ have been also reported with a prevalence higher than $10 \%$ at least in one of the two hystotypes (12). Other novel and rare (i.e., prevalence $<10 \%$ ) gene's alterations have been also reported in NGS studies on ATC $(14,15)$ but their tumorigenic driving role is still undefined.

Tumor associated macrophages (TAMs) have been described in human cancer and their presence in tumoral tissues has been correlated with an advanced stage of the disease and a worse prognosis (16-18). A high density of TAM has been previously reported also in thyroid carcinomas with a higher density in ATC and PDTC with respect to well differentiated papillary thyroid cancer (PTC) $(19,20)$.

The aim of the present study was to verify if, in the clinical practice, ATC and PDTC diagnosed by our pathologist according to the WHO (21) and Turin classification (6) respectively, show a different clinical-pathological profile at diagnosis, a different oncogenic profile, and a different pattern of macrophage infiltration. The correlation between the somatic alterations and the survival time of the patients has been also explored.

\section{Materials and methods}

Patients and tissue samples. Forty-two patients affected with ATC $(n=21)$ or PDTC $(n=21)$ were diagnosed and followed at the Endocrine Unit of the Department of Clinical and Experimental Medicine, University Hospital of Pisa (Pisa, Italy), from 2006 to 2016 . We analyzed the thyroid tumoral DNA from all included patients. Thirty-one tissues were obtained at surgery, immediately frozen in liquid nitrogen and stored at $-80^{\circ} \mathrm{C}$ until extraction; 4 samples derived from formalin fixed paraffin embedded (FFPE) tissues. Seven samples were derived from fine-needle aspiration (FNA) biopsies; FNA was performed under echo guidance by a skilled endocrinologist and using a 23 -gauge needle attached to a $10-\mathrm{ml}$ syringe. The aspirated material was used to prepare slides for cytological analysis. The left over cells were recovered in an appropriate buffer for DNA extraction and stored at $-80^{\circ}$. In 6 cases normal thyroid tissue and/or blood was available. Clinical data were recorded in a computerized data base and analyzed according to the presence of genetic alterations.

A signed informed consent was obtained from all patients and the study received the approval of the Institutional Reviewing Board.

Pathological diagnosis. Patients underwent total or subtotal thyroidectomy at the Department of Surgery of the University of Pisa, Italy. A pre-surgical cytological diagnosis of ATC or PDTC was available for all patients. Hematoxylin-eosin stained sections of all patients from the archives of the section of Pathology of the University of Pisa were re-evaluated independently by two pathologists (C.U. and F.B.). According to the 'Turin proposal' (6) PDTC have been defined as a neoplasm derived from follicular epithelial cells with specific characteristics, that are: i) a solid/trabecular/insular growth pattern; ii) the absence of conventional nuclear features of PTC; and iii) the presence of other features, such as convoluted nuclei, mitotic activity ( $\geq 3$ mitoses per $10 \mathrm{HPF}$ ), or necrosis. Compared to ATC, PDTC shows a monotonous cell population and lack of significant pleomorphism or marked atypia of the nuclei. Moreover, necrosis is typically less prominent in PDTC, usually in focal areas, as compared with the geographic distribution in ATC. Since the immunophenotype is fundamental for the distinction between ATC and PDTC, the immunohistochemistry (IHC) for keratins, thyroglobulin (Tg) and thyroid transcription factor-1 (TTF-1) was performed following standard procedures (22) and the diagnosis took into account the evidence that keratins, Tg and TTF-1 are strongly expressed in well-differentiated carcinomas but typically less intense in PDTC, with Tg and TTF-1 that are completely lost in ATC, that retain only focal and weak expression of keratins.

Genomic DNA and RNA extraction, PCR amplification and sequencing. About $30-40 \mathrm{mg}$ of frozen tissue and the left over cells of FNA were used for genomic DNA extraction using the Maxwell ${ }^{\circledR} 16$ Tissue DNA Purification Kit (Promega Corporation, Madison, WI, USA). Genomic DNA was eluted in $300 \mu \mathrm{l}$ of water. For FFPE tissues, one or two $5 \mu \mathrm{m}$ sections were deparaffinized and digested with proteinase K overnight. DNA extraction was performed using the Maxwell ${ }^{\circledR} 16$ FFPE Tissue LEV DNA Purification Kit (Promega Corporation). DNA was finally diluted in 50-100 $\mu \mathrm{l}$ of water. DNA concentration was measured using an UV spectrophotometer (SmartSpec Plus Spectrophotometer; Bio-Rad Laboratories, Inc., Hercules, CA, USA), and $30 \mathrm{ng}$ of genomic DNA was used for PCR in a final volume of $20 \mu \mathrm{l}$ using a master mix (Kapa2G fast hs ready mix; Resnova S.r.l., Genzano di Roma, Italy).

Primers and PCR conditions were specially defined to the purpose of the present study (Table I). The amplified DNA was analyzed on $2 \%$ agarose gels and purified using a commercial kit (Jetquick purification kit; Celbio, Milan, Italy). Sequence analysis was performed with an automated system employing fluorescent dye terminators (ABI Prism 3110; PerkinElmer, Inc., Waltham, MA, USA). Hot spot positions of NRAS, HRAS, KRAS, BRAF, AKT, PIK3CA, PTEN, TP53, and TERT oncogenes have been sequenced in this study.

Cloning of the deletions. The PCR product of the sample characterized by the presence of heterozigous deletions has been subjected to direct cloning by using the TA cloning Kit (TOPO ${ }^{\circledR}$-TA cloning; Invitrogen; Thermo Fisher Scientific, Inc.) according to the instruction of the manufacturer.

IHC for CD68. In order to investigate the presence of macrophage in tumoral samples, CD68 expression was evaluated by IHC on FFPE tumor sections using mouse monoclonal anti-human antibody (Ventana Medical Systems, Inc., Tucson, AZ, USA). Sections were stained using the Ventana automated slide stainer (Ventana Medical Systems, Inc.). Expression of CD68 was evaluated independently by two of the authors (C.U. and F.B.) who were both blinded to the clinicopathologic data. Discrepancies between the two observers were discussed with a third pathologist (L.T.). The authors evaluated the CD68 expression in macrophages. The samples were evaluated as positive if staining was present. The intensity of the staining was evaluated in 5 high power fields (x40 magnification) and 


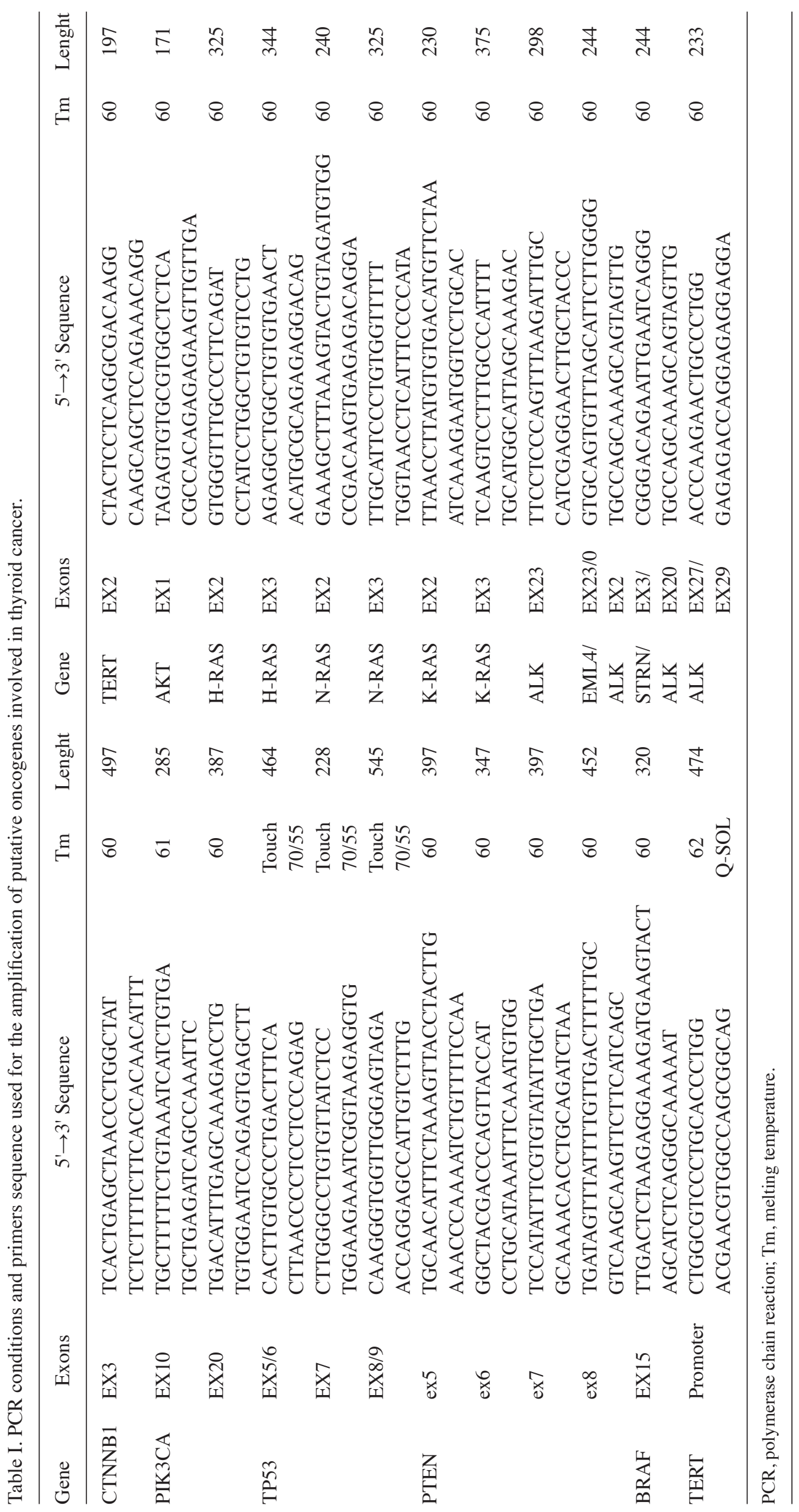


the ratio between the number of CD68 macrophages positive cells and the number of tumoral cells of the samples (i.e., 1:1 and 1:10) was used to create a graduated score: Low (1:100, 1:200), Medium (1:50, 1:20), and High (1:1, 1:2, 1:3, 1:5).

Statistical analysis. The statistical analysis was performed with the Chi-square test according to the studied variables. Survival curves were analyzed using the Kaplan-Meier method and the statistical significance was assessed by log-rank test. $\mathrm{P}<0.05$ was considered to indicate a statistically significant difference.

\section{Results}

Clinico-pathological features of the studied patients. The mean age at diagnosis of the 21 ATC patients (10 males, 11 females) was 65.7 years (median 67, range 45-84). Clinico-pathological data were available in $17 / 21$ patients. According to the TNM classification (23), 2 patients had a stage IVA disease (T4aN0-1M0), 4 a stage IVB (T4bN0-1M0) and 11 a stage IVC (T1-4N0-1M1). Among the 21 patients, 4 were lost at follow-up, 2 were free of disease, 1 had persistent disease and 14 were dead for the tumor with a median survival time of 6 months.

The mean age at diagnosis of the 21 PDTC patients (9 males, 12 females) was 60.2 years (median 58, range 41-83). Clinico-pathological data were available in 17/21 patients; According to the TNM classification (23), 2 patients had a stage II disease (T2NOM0), 1 a stage III (T1-3N0-1M0), 11 a stage IVA (T4aN0-1M0) and 1 a stage IVC (T1-4N0-1M1). For 2 patients that were not surgically treated at our hospital, the presence of node and distant metastases at diagnosis was not known. Among the 21 patients, 4 were lost at follow-up, 4 had persistent disease and 13 were dead for the tumor and the median survival was 47 months.

When comparing the clinical and pathological features of the 2 groups we found that, although not significantly, ATC patients were older than PDTC patients (median 67 vs. 58 years, respectively). Moreover ATC patients had distant metastases at diagnosis much more frequently than PDTC patients (stage IVC $11 / 17$ vs. 1/17, respectively). Finally, although there was no difference in the final outcome in terms of deaths, the median time of survival was significantly shorter in ATC than in PDTC (median survival time 6 vs. 47 months, respectively; $\mathrm{P}=0.0014$ ).

Analysis of somatic mutations. All cases, 21 ATC and 21 PDTC, were screened for gene alterations in 10 selected genes. Overall 44 somatic alterations (40 point mutations and 4 deletions/insertions) were identified in the whole series. Table II shows detailed information on the type of mutations and their prevalence in our series.

In the whole series $25 / 42(59.5 \%)$ cases were found to harbor at least 1 somatic alteration (Fig. 1A) and 17/42 (40.5\%) were found to be negative for the tested mutations. Moreover, in this series, multiple alterations in the same sample were found in several cases $(n=13)$. In the ATC series (Fig. 1B) a total of $15 / 21(71.4 \%)$ cases were found to harbor at least 1 somatic alteration and $6 / 21(28.6 \%)$ were found to be negative for the tested mutations. In this group, mutations of TP53 were the most frequently found (10/21, 47.6\%). In addition to TP53 mutations, we found mutations in TERT $(9 / 21,42.8 \%)$, PTEN (4/21, 19\%), BRAF (4/21, 19\%), N-RAS (2/21, 9.5\%), and PIK3CA $(1 / 21,4.7 \%)$. In the PDTC series a total of $10 / 21$ $(47.6 \%)$ cases were found to harbor at least 1 somatic alteration and 11/21 (52.4\%) were found to be negative for the tested mutations. Mutations were found in TERT (7/21, 33.3\%), BRAF (4/23, 19\%), N-RAS (1/23, 4.7\%), AKT (1/23, 4.7\%) and PIK3CA (1/23, 4.7\%) (Fig. 1C). No mutations were found in TP53 tumor suppressor gene in our PDTC.

Four ATC cases, already included in the above mentioned mutated cases, harbored complex alterations. Two of them were found in the PTEN tumor suppressor gene, the first in exon 5 that was characterized by a 1 bp interstitial deletion (c.355delG, p.V119Lfs*15; COSM28903) and a missense mutation at codon 97 (Q97R) on the same allele; the second was in exon 7 (c.741_742insA, p.P248fs*5; COSM4986). In 2 cases we found deletions in the TP53 tumor suppressor gene: an in frame 3 bp deletion in the exon 6 (c.572_574delCTC, P191delP, COSM111721) and a never reported deletion in exon 7 (c.702_703 del AC, p. H233 fs*1).

When we compared the prevalence of somatic mutations in the group of ATC and PDTC cases we found that TP53 mutations, as well as PTEN mutations, were present in ATC but not in PDTC. Moreover, it is worth to note that complex mutations were found only in ATC. At variance, no major differences were observed in the prevalence of other gene alterations between the two tumoral histotypes. Finally, the prevalence of heterogeneity (i.e., cases with $>2$ mutations) was significantly higher in the ATC group $(6 / 21,28.6 \%)$ than in the PDTC group $(1 / 21,4.7 \%)(\mathrm{P}=0.03)$.

The presence of a germline mutation was excluded in 6/6 cases whose normal thyroid tissue and/or blood was available.

Analysis of macrophagic infiltration in ATC and PDTC. Analysis of TAM was performed by evaluating the CD68 expression by IHC. Twenty-six cases (15 ATC and 11 PDTC) whose paraffin embedded tissues were available, were evaluated. All samples turned out to be positive for CD68 although at a variable degree (Fig. 2A-C). In particular among PDTC the CD68 staining was high in 2 cases, and low in the remaining 9 cases; among ATC the CD68 staining was high in 11 cases, medium in 3 cases and low in 1 case. When comparing the intensity of CD68 staining in ATC and PDTC we found that ATC were characterized by a higher macrophagic infiltration ( $\mathrm{P}=0.0005)$ (Fig. 2D). Moreover, in ATC the intensity of CD68 staining correlated with the presence of TP53 mutations even if, although very close, this correlation did not reach a formal statistical significance (Fig. 2E, $\mathrm{P}=0.05$ ).

Correlation of somatic alterations with the outcome. We correlated the presence of the somatic alterations with the outcome of PDTC and ATC patients. When considering the whole group, data on the outcome of patients were available in 34 cases (17 PDTC and 17 ATC). Among them, 28 patients were died for the tumor, 4 have persistent disease and 2 have been cured and still alive. It is worth to note that these latter 
Table II. Genetic alterations in our series $(n=42)$ of ATC and PDTC cases.

\begin{tabular}{|c|c|c|c|c|c|}
\hline Gene & Mutation & Exon & $\begin{array}{l}\text { Mutated cases } \\
\text { in ATC }(n=21)\end{array}$ & $\begin{array}{l}\text { Mutated cases } \\
\text { in PDTC }(n=21)\end{array}$ & $\mathrm{P}\left(\chi^{2}\right)$ \\
\hline NRAS & Q61R & 3 & $2(9.5 \%)$ & $1(4.7 \%)$ & NS \\
\hline BRAF & V600E & 15 & $4(19 \%)$ & $4(19 \%)$ & NS \\
\hline AKT & E17K & 1 & 0 & $1(4.7 \%)$ & NS \\
\hline \multirow[t]{10}{*}{ TP53 } & C176Y & 5 & $1(4.75 \%)$ & 0 & 0.0003 \\
\hline & $\mathrm{H} 179 \mathrm{R}$ & & $1(4.75 \%)$ & 0 & \\
\hline & c.572_574delCTC, P191delP & 6 & $1(4.75 \%)$ & 0 & \\
\hline & M246L & 7 & $1(4.75 \%)$ & 0 & \\
\hline & R248W & & $1(4.75 \%)$ & 0 & \\
\hline & c.702_703 del AC, p. H233 fs*1 & & $1(4.75 \%)$ & 0 & \\
\hline & $\mathrm{R} 267 \mathrm{~W}$ & 8 & $1(4.75 \%)$ & 0 & \\
\hline & R273C & & $1(4.75 \%)$ & 0 & \\
\hline & $\mathrm{R} 273 \mathrm{H}$ & & $1(4.75 \%)$ & 0 & \\
\hline & K320N & 9 & $1(4.75 \%)$ & 0 & \\
\hline \multirow[t]{2}{*}{ PIK3CA } & E545K & 10 & $1(4.75 \%)$ & 0 & NS \\
\hline & H1047R & 20 & 0 & $1(4.7 \%)$ & \\
\hline \multirow[t]{5}{*}{ PTEN } & c.355delG, p. V119Lfs*15 & & & & \\
\hline & Q97R & 5 & $1(4.75 \%)$ & 0 & 0.03 \\
\hline & $\mathrm{D} 115 \mathrm{~N}$ & & $1(4.75 \%)$ & 0 & \\
\hline & S170I & 6 & $1(4.75 \%)$ & 0 & \\
\hline & c.741_742insA, p. P248fs*5 & 7 & $1(4.75 \%)$ & 0 & \\
\hline \multirow[t]{3}{*}{ TERT } & $\mathrm{C} 250 \mathrm{~T}$ & Promoter & $1(4.75 \%)$ & 0 & NS \\
\hline & $\mathrm{C} 228 \mathrm{~T}$ & & $7(33 \%)$ & $9(43 \%)$ & \\
\hline & P376A & 2 & 0 & $1(4.7 \%)$ & \\
\hline \multirow{3}{*}{$\begin{array}{l}\text { ALK } \\
\text { n. mutations }\end{array}$} & EML4/ALK rearrangement & $14 / 20$ & 0 & $1 / 9(11.1 \%)$ & NS \\
\hline & $>2$ & & $6(28.6 \%)$ & $1(4.7 \%)$ & 0.03 \\
\hline & $\leq 2$ & & $15(71.4 \%)$ & $20(95.3 \%)$ & \\
\hline
\end{tabular}

ATC, anaplastic thyroid cancer; PDTC, poorly differentiated thyroid cancer; NS, not significant.

patients were both affected by ATC (confirmed by 3 different pathologists) but negative for all studied mutations.

In our series 19/19 (100\%) patients with at least 1 somatic mutation died for the disease (ATC $n=12$, PDTC $n=7$ ) while in the group of non mutated cases only $9 / 15(60 \%)$ were died (ATC $n=3$, PDTC $n=6$ ). The remaining non mutated cases $6 / 15$ $(40 \%)$ patients were either cured $(2 / 15,13.3 \%)(\mathrm{ATC} n=2)$ or affected by a persistent disease $(4 / 15,26.7 \%)$ (PDTC $n=4)$. When analyzing the overall survival length we observed that in the whole series a shorter survival was significantly correlated with the presence of more than 2 gene mutations in the same tissue (Fig. 3A) Moreover, the absence of TP53 mutation appears to be a good prognostic factor for a longer survival (Fig. 3B).

\section{Discussion}

ATC and PDTC are rare dedifferentiated thyroid tumors that are supposed to derive either by pre-existing well differentiated thyroid carcinomas (24) or to originate directly from normal follicular thyroid cells (25). Both of them are very aggressive but the ATC have a worse prognosis in terms of shortness of survival $(4,5)$ and also in the modality of death which is by suffocation in the majority of cases (26). The differential diagnosis is very important at clinical level since the medical decisions to be taken are very depending from histology mainly because PDTC can be treated with tyrosine kinase inhibitors, such as sorafenib and lenvatinib $(27,28)$, while these drugs are not yet approved for the treatment of ATC.

In the present series we found that our ATC and PDTC, which were classified and distinguished according to the WHO and Turin classifications, respectively, were represented by two groups of patients with a different clinical and pathological profile already at the time of the diagnosis. Although mean age of patients was already $>60$ in both groups, the PDTC group showed a younger age at diagnosis than ATC (median age 58 vs. 67 years). Moreover, the stage IVC at diagnosis, the only one comparable since ATC are all stage IV by definition, was more frequent in ATC $(11 / 17 ; 64.7 \%)$ than in PDTC $(1 / 17 ; 5.9 \%)$. Finally, although the rate of death was high in both in ATC and PDTC (i.e., 82.3 vs. $76.5 \%$, respectively), 


\section{A Whole series}

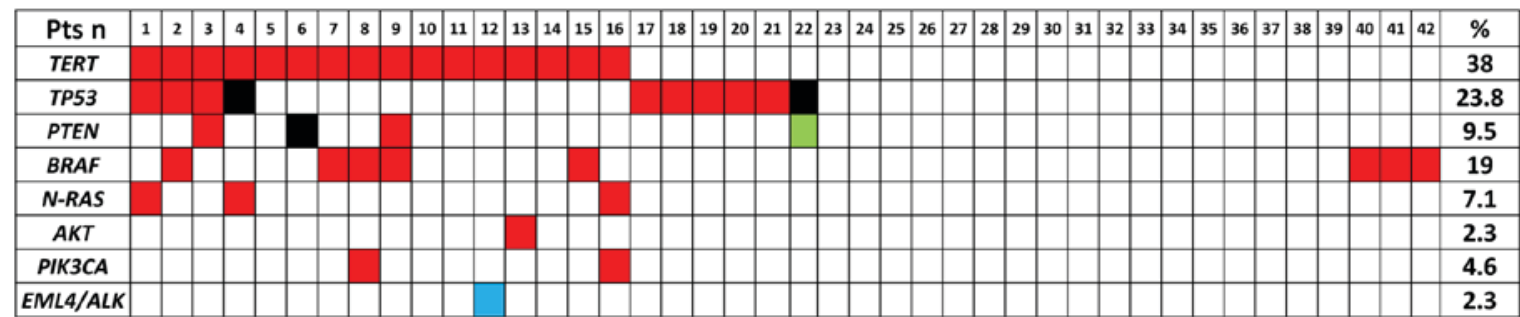

\section{B ATC}

\begin{tabular}{|c|c|c|c|c|c|c|c|c|c|c|c|c|c|c|c|}
\hline Pts n & \begin{tabular}{l|l}
1 & 2 \\
\end{tabular} & 3 & \begin{tabular}{|l|l}
4 & 5 \\
\end{tabular} & \begin{tabular}{|l|l|}
5 & 6 \\
\end{tabular} & \begin{tabular}{|l|l|}
7 & 8 \\
\end{tabular} & \begin{tabular}{|l|l|}
8 & 9 \\
\end{tabular} & \begin{tabular}{|l|l|}
17 & 18 \\
\end{tabular} & 18 | 19 & \begin{tabular}{|l|l|}
20 & 21 \\
\end{tabular} & 122 & \begin{tabular}{|l|l|}
23 & 24 \\
\end{tabular} & 25 & 26 & \begin{tabular}{|l|l|}
27 & 2 \\
\end{tabular} & $\%$ \\
\hline TERT & & & & & & & & & & & & & & & 42.8 \\
\hline TP53 & & & & & & & & & & & & & & & 47.6 \\
\hline PTEN & & & & & & & & & & & & & & & 19 \\
\hline BRAF & & & & & & & & & & & & & & & 19 \\
\hline$N-R A S$ & & & & & & & & & & & & & & & 9.5 \\
\hline AKT & & & & & & & & & & & & & & & 0 \\
\hline PIK3CA & & & & & & & & & & & & & & & 4.7 \\
\hline$E M L 4 / A L K$ & & & & & & & & & & & & & & & 0 \\
\hline
\end{tabular}

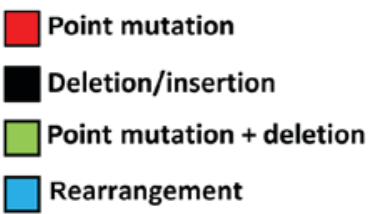

C PDTC

\begin{tabular}{|c|c|c|c|c|c|c|c|c|c|c|c|c|c|c|}
\hline Pts $n$ & \begin{tabular}{|l|l|l|}
10 & 11 & 12 \\
\end{tabular} & \begin{tabular}{|l|l|}
13 & 14 \\
\end{tabular} & 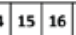 & \begin{tabular}{|l|l|}
29 & 30 \\
\end{tabular} & 31 & 323 & \begin{tabular}{ll|l}
33 & 34 \\
\end{tabular} & \begin{tabular}{|l|l}
35 & 3 \\
\end{tabular} & \begin{tabular}{l|l}
36 & 37 \\
\end{tabular} & 38 & \begin{tabular}{|l|l|}
39 & $4 c$ \\
\end{tabular} & \begin{tabular}{l|l|}
10 & 42
\end{tabular} & 42 & $\%$ \\
\hline TERT & & & & & & & & & & & & & & 33.3 \\
\hline TP53 & & & & & & & & & & & & & & 0 \\
\hline PTEN & & & & & & & & & & & & & & 0 \\
\hline BRAF & & & & & & & & & & & & & & 19 \\
\hline$N-R A S$ & & & & & & & & & & & & & & 4.7 \\
\hline$A K T$ & & & & & & & & & & & & & & 4.7 \\
\hline PIKЗСА & & & & & & & & & & & & & & 4.7 \\
\hline$E M L 4 / A L K$ & & & & & & & & & & & & & & 4.7 \\
\hline
\end{tabular}

Figure 1. Oncogene alterations in ATC and PDTC. Red squares, point mutations; black squares, complex alterations; Green square, complex alteration plus point mutation; blu square, gene rearrangement. (A) Mutations in the whole series (21 ATC and 21 PDTC); (B) Mutations in the ATC subgroup; (C) Mutations in the PDTC subgroup. TC, anaplastic thyroid cancer; PDTC, poorly differentiated thyroid cancer.

the length of survival of the dead patients was significantly longer in PDTC (median 47 months) than in ATC (median 6 months). Our findings are in keeping with those reported in the literature since it is known that PDTC, although lethal in the majority of cases, have a slower aggressiveness than ATC and are characterized by a lower stage at presentation and longer follow up $(2,5)$. In our opinion, the fact that in our series the two groups maintain these clinical and biological differences among them indirectly supports the truthfulness of the histological classifications used by our pathologists.

As far as the molecular profiles of ATC and PDTC are concerned, both traditional techniques and NGS confirmed a high prevalence of mutations of 'classical' thyroid tumors related oncogenes and/or tumor suppression genes such as TP53, TERT and BRAF $(12,14,15,29,30)$. We analysed the most frequent genetic alterations involved in ATC and PDTC tumorigenesis and in particular we analysed those alterations that were found to be prevalent at least in more than $10 \%$ of cases (12). Also in our series, TERT and TP53 mutations are the most frequent in ATC histotype followed by $B R A F$ $V 600 E$ and $N-R A S$ mutations with a prevalence that is almost the half of that found in the Landa et al study. A likely reason to explain this difference can be found in the fact that the NGS method used by Landa et al is much more sensitive than the Sanger sequencing method used by us and since, as we also observed, ATC tissues are reach of macrophages, their presence can further reduce the sensitivity in detecting gene mutations. However, at variance, the prevalence of PTEN alterations in our ATC series (19\%) was very similar to that found by Landa et al (18\%) thus raising the question of whether we had a real problem of low sensitivity or whether our series of ATC were somehow different from those analysed by Landa et al, although in both series the WHO 2004 classification was used.

Regarding the PDTC gene alterations we confirmed that also in our series TERT alterations were the most frequent followed by BRAFV600E and N-RAS with a prevalence that was again about half of that found by Landa et al. A peculiar data is that we did not find any alteration of TP53 and PTEN in our series of PDTC and this finding is in contrast with data reported by NGS $(12)$ and other studies $(29,31,32)$ that showed the presence of TP53 mutations in $8-28 \%$ of PDTC cases. One possible explanation to justify this difference is that the differential diagnosis between PDTC and ATC is dependent from pathologists and type of classifications. Italian PDTC cases were diagnosed taking into consideration the Turin classification and Landa's PDTC cases were classified taking into consideration either the Turin classification or their own (MSK) criteria. As shown in the Landa's paper (12) the genetic profile of PDTC tumor is different according to the different system of classification and this could also justify the different prevalence of TP53 and PTEN mutations in our cases. 

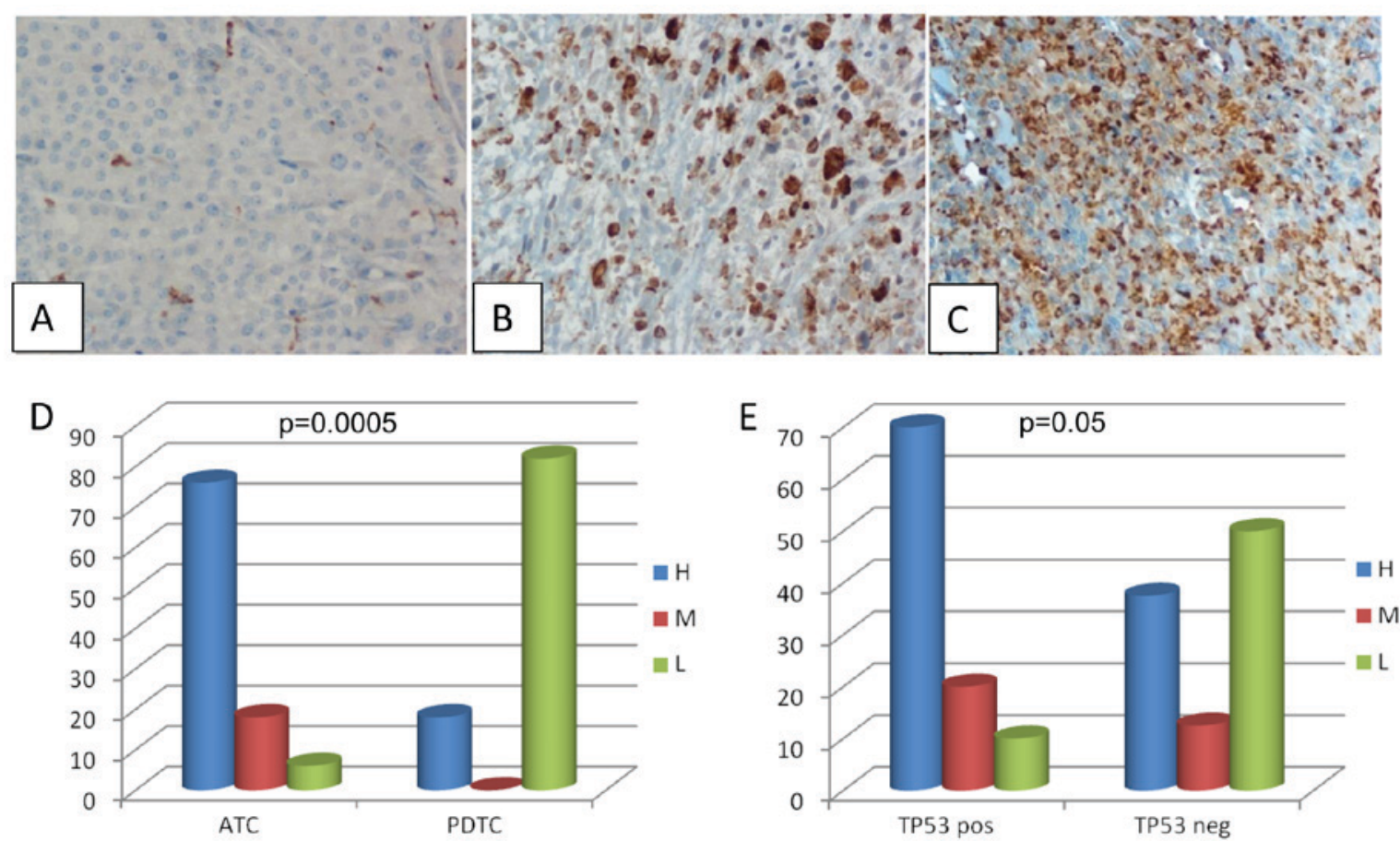

Figure 2. TAM infiltration in ATC and PDTC. (A-C) Immunohistochemical detection of CD68 positivity showing different degrees of positivity (panel A, low; panel B, medium; panel C, high); (D) CD68 positive cases: comparison of the degree of positivity in the ATC and PDTC subgroups; (E) CD68 positive cases: Comparison of the degree of positivity in the TP53 positive and negative cases. TC, anaplastic thyroid cancer; PDTC, poorly differentiated thyroid cancer.
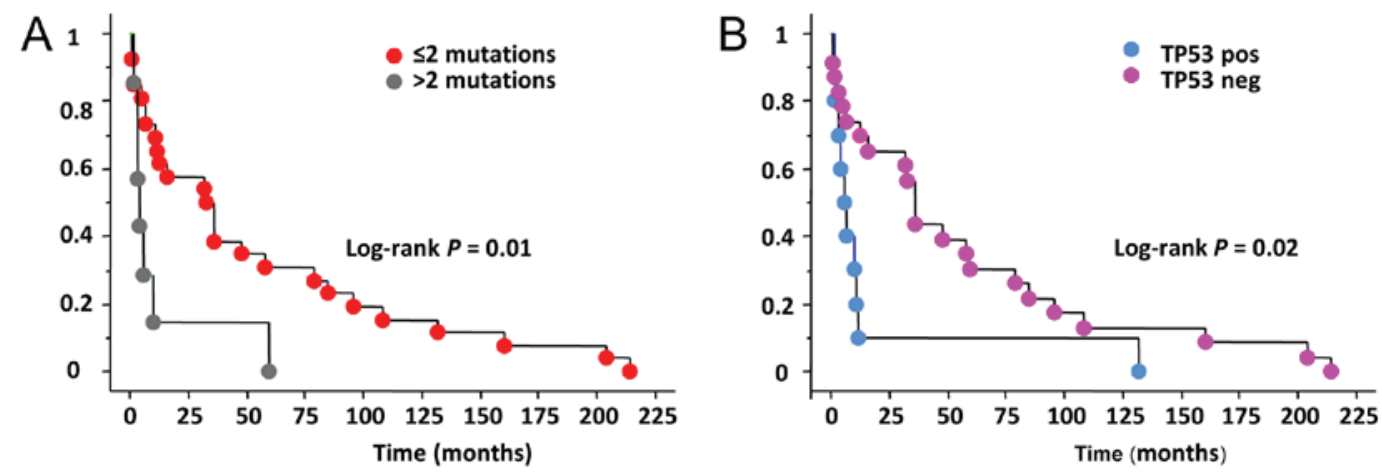

Figure 3. Kaplan Meyer Survival curves in ATC and PDTC. (A) Comparison between the survival curves according to the number of mutations; (B) Comparison between the survival curves of TP53 positive and negative cases. TC, anaplastic thyroid cancer; PDTC, poorly differentiated thyroid cancer.

Another difference that we observed between ATC and PDTC genetic profile is related to the presence of both a higher number of mutated cases and a higher number of heterogeneous cases with $>2$ mutations in the same tumor. This finding is in keeping with that of Landa and colleagues that also showed that the median number of Single Nucleotide Variants in ATC is higher than in PDTC (12). Cases with a number of mutation $>2$ showed a worse prognosis in our overall series and the evidence that the number of cases with multiple mutations is higher in ATC confirmed that these are much more aggressive than PDTC. This finding is in line with the evidence that human tumors with a higher degree of heterogeneity, such as pancreatic tumors, are more aggressive $(33,34)$. As far as the correlation between the mutations and the clinical course of the disease was concerned, we found that patients with tumors positive for TP53 mutation, that must be underlined were all ATC, had a shorter survival time, as observed also in other human cancer (35-37). However, their final outcome (i.e. dead vs. alive patients) did not show any statistically significant differences in the TP53 positive vs. negative cases. It is worth noting that the 2 cured patients, although affected by well ascertained ATC, were both negative for any mutation.

A clear distinction between ATC and PDTC has also been highlighted by differences in the level of TAM infiltrating the tumors. In fact, although all cases, both ATC and PDTC, turned out to be positive for CD68 immunostaining, a higher percentage of CD68 positive cells was demonstrated in ATC with respect to PDTC. The correlation between tumor progression and presence of TAM has been previously reported in thyroid tumors (19) and a correlation between TAM, tumor vascularization and metastases has been demonstrated also in other human tumors $(16,38)$. A significant correlation of higher levels of TAM expression has been observed in our TP53 positive cases, that were only ATC, in keeping with 
the observation that mutations in this gene may support a pro-inflammatory tumor microenvironment promoting tumor malignancy (39). To our knowledge this is the first study demonstrating a correlation between TP53 and presence and intensity of TAM in ATC.

We are aware of the limits of this study due to the low number of cases and the incompleteness of some data but, unfortunately (or fortunately) these tumors are very rare also in referral centers like ours and the collection of the samples was in 10 years during which the strategies of therapy (i.e., surgery vs. non surgery) and follow up (i.e., periodical active controls vs. palliative care) of these patients have been changing and impacting on the outcome of these patients. Only a multicentric study with shared protocols of therapy and follow up could overcome this problem.

Despite these considerations, according to our results and taking into account the limit above mentioned, we can conclude that ATC and PDTC, although both of them still lethal, are different tumors not only because characterized by a different clinical and pathological profile at diagnosis but also, at least in our series, because they show a different molecular profile in terms of type and number of mutations and also a different spectrum of positivity for TAM. New targeted therapies for both, and in particular for PDTC, can provide a chance of prolonged survival. Finally, according to our results, the WHO classification for ATC and the Turin classification for PDTC are likely those that better distinguish the two types of very aggressive thyroid cancer.

\section{Acknowledgements}

This study was supported by the Associazione Italiana Ricerca sul Cancro (AIRC, Investigator grant 2014, project code 15431).

\section{References}

1. Khairy G: Anaplastic transformation of differentiated thyroid carcinoma. Int J Health Sci (Qassim) 3: 93-96, 2009.

2. Yu MG, Rivera J and Jimeno C: Poorly differentiated thyroid carcinoma: 10-year experience in a southeast asian population. Endocrinol Metab (Seoul) 32: 288-295, 2017.

3. Molinaro E, Romei C, Biagini A, Sabini E, Agate L, Mazzeo S, Materazzi G, Sellari-Franceschini S, Ribechini A, Torregrossa L, et al: Anaplastic thyroid carcinoma: From clinicopathology to genetics and advanced therapies. Nat Rev Endocrinol 13: 644-660, 2017.

4. Nagaiah G, Hossain A, Mooney CJ, Parmentier J and Remick SC: Anaplastic thyroid cancer: A review of epidemiology, pathogenesis and treatment. J Oncol 2011: 542358, 2011.

5. Siironen P, Hagström J, Mäenpää HO, Louhimo J, Heikkilä A, Heiskanen I, Arola J and Haglund C: Anaplastic and poorly differentiated thyroid carcinoma: Therapeutic strategies and treatment outcome of 52 consecutive patients. Oncology 79: 400-408, 2010.

6. Volante M, Collini P, Nikiforov YE, Sakamoto A, Kakudo K, Katoh R, Lloyd RV, LiVolsi VA, Papotti M, Sobrinho-Simoes M, et al: Poorly differentiated thyroid carcinoma: The Turin proposal for the use of uniform diagnostic criteria and an algorithmic diagnostic approach. Am J Surg Pathol 31: 1256-1264, 2007.

7. Volante M, Bussolati G and Papotti M: The story of poorly differentiated thyroid carcinoma: From Langhans' description to the Turin proposal via Juan Rosai. Semin Diagn Pathol 33: 277-283, 2016.

8. Volante M and Papotti M: Poorly differentiated thyroid carcinoma: 5 years after the 2004 WHO classification of endocrine tumours. Endocr Pathol 21: 1-6, 2010.
9. Asioli S, Erickson LA, Righi A, Jin L, Volante M, Jenkins S, Papotti M, Bussolati G and Lloyd RV: Poorly differentiated carcinoma of the thyroid: Validation of the Turin proposal and analysis of IMP3 expression. Mod Pathol 23: 1269-1278, 2010.

10. Smallridge RC and Copland JA: Anaplastic thyroid carcinoma: Pathogenesis and emerging therapies. Clin Oncol (R Coll Radiol) 22: 486-497, 2010.

11. Shi X, Liu R, Qu S, Zhu G, Bishop J, Liu X, Sun H, Shan Z, Wang E, Luo Y, et al: Association of TERT promoter mutation 1,295,228 C>T with BRAF V600E mutation, older patient age and distant metastasis in anaplastic thyroid cancer. J Clin Endocrinol Metab 100: E632-E637, 2015.

12. Landa I, Ibrahimpasic T, Boucai L, Sinha R, Knauf JA, Shah RH, Dogan S, Ricarte-Filho JC, Krishnamoorthy GP, Xu B, et al: Genomic and transcriptomic hallmarks of poorly differentiated and anaplastic thyroid cancers. J Clin Invest 126: 1052-1066, 2016.

13. Nikiforov YE: Genetic alterations involved in the transition from well-differentiated to poorly differentiated and anaplastic thyroid carcinomas. Endocr Pathol 15: 319-327, 2004.

14. KunstmanJW,JuhlinCC,GohG,BrownTC,Stenman A,Healy JM, Rubinstein JC, Choi M, Kiss N, Nelson-Williams C, et al: Characterization of the mutational landscape of anaplastic thyroid cancer via whole-exome sequencing. Hum Mol Genet 24: 2318-2329, 2015

15. Jeon MJ, Chun SM, Kim D, Kwon H, Jang EK, Kim TY, Kim WB, Shong YK, Jang SJ, Song DE and Kim WG: Genomic alterations of anaplastic thyroid carcinoma detected by targeted massive parallel sequencing in a BRAF (V600E) mutation-prevalent area. Thyroid 26: 683-690, 2016.

16. Lissbrant IF, Stattin P, Wikstrom P, Damber JE, Egevad L and Bergh A: Tumor associated macrophages in human prostate cancer: Relation to clinicopathological variables and survival. Int J Oncol 17: 445-451, 2000.

17. Ohno S, Ohno Y, Suzuki N, Kamei T, Koike K, Inagawa H, Kohchi C, Soma G and Inoue M: Correlation of histological localization of tumor-associated macrophages with clinicopathological features in endometrial cancer. Anticancer Res 24: 3335-3342, 2004.

18. Hanada T, Nakagawa M, Emoto A, Nomura T, Nasu N and Nomura Y: Prognostic value of tumor-associated macrophage count in human bladder cancer. Int J Urol 7: 263-269, 2000.

19. Ryder M, Ghossein RA, Ricarte-Filho JC, Knauf JA and Fagin JA: Increased density of tumor-associated macrophages is associated with decreased survival in advanced thyroid cancer. Endocr Relat Cancer 15: 1069-1074, 2008.

20. Caillou B, Talbot M, Weyemi U, Pioche-Durieu C, Al Ghuzlan A, Bidart JM, Chouaib S, Schlumberger $M$ and Dupuy C: Tumor-associated macrophages (TAMs) form an interconnected cellular supportive network in anaplastic thyroid carcinoma. PLoS One 6: e22567, 2011.

21. Lloyd RV OR, Klöppel G and Rosai J (eds). WHO Classification of Tumours of Endocrine Organs. 4th edition. WHO, Geneva, 2017.

22. Bejarano PA, Nikiforov YE, Swenson ES and Biddinger PW: Thyroid transcription factor-1, thyroglobulin, cytokeratin 7 , and cytokeratin 20 in thyroid neoplasms. Appl Immunohistochem Mol Morphol 8: 189-194, 2000.

23. Edge SB and Compton CC: The American Joint Committee on Cancer: The 7th edition of the AJCC cancer staging manual and the future of TNM. Ann Surg Oncol 17: 1471-1474, 2010.

24. Wreesmann VB, Ghossein RA, Patel SG, Harris CP, Schnaser EA, Shaha AR, Tuttle RM, Shah JP, Rao PH and Singh B: Genome-wide appraisal of thyroid cancer progression. Am J Pathol 161: 1549-1556, 2002.

25. O'Neill JP and Shaha AR: Anaplastic thyroid cancer. Oral Oncol 49: 702-706, 2013.

26. Shaha AR, Ferlito A, Owen RP, Silver CE, Rodrigo JP, Haigentz M Jr, Mendenhall WM, Rinaldo A and Smallridge RC: Airway issues in anaplastic thyroid carcinoma. Eur Arch Otorhinolaryngol 270: 2579-2583, 2013.

27. Brose MS, Nutting CM, Jarzab B, Elisei R, Siena S, Bastholt L, de la Fouchardiere C, Pacini F, Paschke R, Shong YK, et al: Sorafenib in radioactive iodine-refractory, locally advanced or metastatic differentiated thyroid cancer: A randomised, double-blind, phase 3 trial. Lancet 384: 319-328, 2014.

28. Schlumberger M, Tahara M, Wirth LJ, Robinson B, Brose MS, Elisei R, Habra MA, Newbold K, Shah MH, Hoff AO, et al: Lenvatinib versus placebo in radioiodine-refractory thyroid cancer. N Engl J Med 372: 621-630, 2015. 
29. Donghi R, Longoni A, Pilotti S, Michieli P, Della Porta G and Pierotti MA: Gene p53 mutations are restricted to poorly differentiated and undifferentiated carcinomas of the thyroid gland. J Clin Invest 91: 1753-1760, 1993.

30. Fagin JA, Matsuo K, Karmakar A, Chen DL, Tang SH and Koeffler HP: High prevalence of mutations of the p53 gene in poorly differentiated human thyroid carcinomas. J Clin Invest 91: 179-184, 1993.

31. Ho YS, Tseng SC, Chin TY, Hsieh LL and Lin JD: p53 gene mutation in thyroid carcinoma. Cancer Lett 103: 57-63,1996.

32. Dobashi Y, Sugimura H, Sakamoto A, Mernyei M, Mori M, Oyama T and Machinami R: Stepwise participation of p53 gene mutation during dedifferentiation of human thyroid carcinomas. Diagn Mol Pathol 3: 9-14, 1994.

33. Cros J, Raffenne J, Couvelard A and Pote N: Tumor heterogeneity in pancreatic adenocarcinoma. Pathobiology: Aug 5, 2017 (Epub ahead of print).

34. Riesco-Eizaguirre G and Santisteban P: Endocrine Tumours: Advances in the molecular pathogenesis of thyroid cancer: Lessons from the cancer genome. Eur J Endocrinol 175: R203-R217, 2016.

35. Girgin C, Tarhan H, Hekimgil M, Sezer A and Gürel G: P53 mutations and other prognostic factors of renal cell carcinoma. Urol Int 66: 78-83, 2001.

36. Kim HW, Lee HM, Hwang SH, Ahn SG, Lee KA and Jeong J: Patterns and biologic features of p53 mutation types in korean breast cancer patients. J Breast Cancer 17: 1-7, 2014.
37. Horio Y, Takahashi T, Kuroishi T, Hibi K, Suyama M, Niimi T, Shimokata K, Yamakawa K, Nakamura Y, Ueda R, et al: Prognostic significance of p53 mutations and 3p deletions in primary resected non-small cell lung cancer. Cancer Res 53: 1-4, 1993.

38. Bolat F, Kayaselcuk F, Nursal TZ, Yagmurdur MC, Bal N and Demirhan B: Microvessel density, VEGF expression and tumor-associated macrophages in breast tumors: Correlations with prognostic parameters. J Exp Clin Cancer Res 25: 365-372, 2006.

39. Ubertini V, Norelli G, D'Arcangelo D, Gurtner A, Cesareo E, Baldari S, Gentileschi MP, Piaggio G, Nistico P, Soddu S, et al: Mutant p53 gains new function in promoting inflammatory signals by repression of the secreted interleukin-1 receptor antagonist. Oncogene 34: 2493-2504, 2015.

This work is licensed under a Creative Commons Attribution-NonCommercial-NoDerivatives 4.0 International (CC BY-NC-ND 4.0) License. 